\section{Medicamentos alternativos e novos fármacos}

Os anticoagulantes tradicionalmente usados no tratamento e na prevenção das doenças tromboembólicas são as heparinas e a varfarina. Essas medicações têm limitações importantes, como a estreita janela terapêutica e a alta variação dose-resposta para cada indivíduo, demandando monitorização laboratorial frequente. Os AVK têm muitas interações medicamentosas e podem ser alterados até pela dieta (alimentos ricos em vitamina K), provocando um importante problema, principalmente quando a anticoagulação é indicada por tempo indeterminado. A heparina é utilizada na prática clínica desde a década de $30 .{ }^{(1)}$ Há vários efeitos colaterais relacionados ao seu uso: hemorragias, osteoporose, reações cutâneas, eosinofilia, alopecia, alterações nos testes da função hepática e hipercalcemia ocasional. ${ }^{(2,3)}$ No entanto, um dos mais importantes é a trombocitopenia. ${ }^{(3,4)}$ Devido a essas limitações, foram necessárias pesquisas para o desenvolvimento de novas drogas anticoagulantes. ${ }^{(5)}$

Os novos anticoagulantes são classificados pelo seu mecanismo de ação (Quadro 23). Atualmente, o principal uso desses fármacos alternativos é no tratamento de $\mathrm{TIH}$.

\section{Heparinoides}

0 danaparoide, que é composto pelos sulfatos de heparan, dermatan e condroitina, tem sido extensivamente utilizado na $\mathrm{TlH}$ num amplo contexto de doenças. Apesar da ocorrência de 15\% de reações cruzadas de anticorpos contra a heparina in vivo, raramente causa piora de $\mathrm{TlH}^{\left({ }^{(6)}\right.} \mathrm{O}$ embasamento para seu uso provém de ensaios clínicos abertos e de série de casos. ${ }^{(7)} 0$ danaparoide não está mais disponível nos EUA e Reino Unido, mas, onde está disponível, tem sido largamente utilizado em pacientes com $\mathrm{TlH}^{\left({ }^{(8,9)}\right.}$ Sua monitorização é feita pelos níveis de antifator Xa.

\section{Inibidores diretos da trombina}

A função primordial da trombina é provocar a cisão da molécula de fibrinogênio, originando o polipeptídeo A e o monômero de fibrina. A formação da fibrina segue, em um destino irreversível, para consolidar a rede de fibrina, com ajuda do fator Xll ativado. ${ }^{(10)}$ A trombina, sem função fisiológica, tem que sofrer neutralização, e dessa participam vários mecanismos celulares e humorais. 0s inibidores diretos da trombina bloqueiam a trombina sem a necessidade de cofatores e sem intervir em outros mecanismos pró-trombóticos. Essa ação, independente de cofatores, torna as antitrombinas drogas potentes para evitar a trombose, pois não há individualidade de ação, como ocorre com os coatores, e por ter ação direta, neutraliza a trombina onde ela se encontrar. Os inibidores diretos da trombina têm duas vantagens inquestionáveis em substituição ao complexo antitrombina-heparina: na plaquetopenia induzida por heparina e na deficiência congênita de antitrombina. ${ }^{(4)}$

\section{Hirudina}

A hirudina é um potente e específico inibidor da trombina. ${ }^{(11)}$ Ela consegue se ligar à trombina com alta afinidade e especificidade, formando um complexo não covalente irreversível, inibindo, assim, todas as funções proteolíticas da trombina. ${ }^{(12)}$ Ao contrário da heparina, a hirudina não é inativada pelo fator plaquetário 4 e, como apresenta estrutura química diferente, não existe reação cruzada entra essas drogas. ${ }^{(13)}$ É excretada pelo rim e é necessário o ajuste de doses em pacientes com insuficiência renal. 0 controle da droga é feito pelo TTPa (mantido a $1,5-2,5$ vezes o valor do controle), e as principais desvantagens são o custo e a falta de antídoto para a mesma. ${ }^{(12)}$ Alguns estudos mostram sua maior eficácia do que a enoxaparina na profilaxia de TVP em cirurgias ortopédicas, sem haver diferenças significativas nas complicações, como sangramentos. ${ }^{(14)}$

Várias formas comerciais de hirudina são fabricadas através do emprego de biotecnologia recombinante. ${ }^{(15)}$ A lepirudina é uma hirudina recombinante dissulfatada, estando indicada no tratamento da plaquetopenia induzida por heparina, promovendo uma rápida e sustentada recuperação da contagem de plaquetas, indicando uma ausência de reação cruzada, com risco de sangramento semelhante ao da heparina. 0 efeito adverso mais comum é o sangramento, sendo outros efeitos as reações cutâneas, sepse, pneumonia e aumento das enzimas hepáticas. 0 uso por mais de 5 dias de lepirudina tem sido relacionado à formação de anticorpos lgG antihirudina. Nesses pacientes, há um aumento maior de TTPa, necessitando de uma monitorização mais frequente. ${ }^{(16)}$

Está aprovada na Europa, desde 1997, e nos EUA, desde 1998, para o tratamento da $\mathrm{TIH} .{ }^{(13)}$ 


\section{Argatrobana}

É uma droga sintética que atua de forma reversível, direta e seletiva. Atinge uma rápida eficácia terapêutica antitrombótica, com um mínimo risco de sangramento e rápida restauração da hemostasia no momento da descontinuação. ${ }^{(17)} \mathrm{A}$ argatrobana tem meia-vida curta e, como a ligação com a trombina é reversível, torna-o mais seguro e com menor risco de sangramento em relação à hirudina recombinante. ${ }^{(18)}$ É necessária precaução em pacientes portadores de disfunção hepática, já que essa é a via de excreção. Não há necessidade de ajuste de dose em portadores de insuficiência renal. ${ }^{(19)} \mathrm{Em}$ junho de 2000, o Food and Drug Administration aprovou a droga para uso na trombocitopenia provocada por heparina. Esse fármaco foi utilizado, na maioria dos casos, em pacientes com cardiopatia isquêmica agudizada, tendo sido aplicado em pacientes com TEV em pequenas séries de casos. ${ }^{(20)}$ A sua monitorização é feita pelo TTPa, mantendo-o entre $1,5-3$ vezes o controle (não excedendo 100 s). ${ }^{(21)}$

\section{Ximelagatrana}

Este fármaco foi uma esperança promissora como um inibidor direto da trombina, pois era o primeiro que poderia ser administrado por via oral. (22) Seria uma ótima opção terapêutica para pacientes que necessitariam de anticoagulação prolongada por prescindir de monitorização. Entretanto, a alta incidência de hepatotoxici- dade deixou seu uso com restrições importantes. (23) Em 2004, o Food and Drug Administration desaprovou o uso da medicação devido aos seus importantes efeitos colaterais, sobretudo relacionados à hepatotoxicidade. ${ }^{(24)}$ No Brasil, essa medicação teve seu uso suspenso antes da comercialização.

\section{Dabigatrana}

Recentemente, foi lançado no mercado brasileiro o etexilato de dabigatrana, um inibidor reversível do sítio ativo da trombina. É um agente para uso oral que prescinde de monitorização laboratorial. Cerca de $80 \%$ da droga é excretada via renal, não sendo recomendável seu uso em pacientes com insuficiência renal moderada a grave. Esse fármaco foi testado em ensaios clínicos de fase 111 na prevenção de TEV em cirurgias ortopédicas, sendo comparado à enoxaparina. Deve ser iniciado de 1-4 h após o término da cirurgia na dose de $110 \mathrm{mg}$. Deve ser aumentado para $220 \mathrm{mg}$ no dia subsequente se não houver sangramento, mantendo-se sua administração até o total de 10 dias. Na maioria dos estudos, não houve diferenças estatisticamente significativas nos desfechos de incidência de TEV, sangramento e mortalidade. ${ }^{(25-27)}$ Pode ser uma alternativa às HBPM na prevenção de TEV em pacientes ortopédicos. Um recente ensaio clínico randomizado de não inferioridade comparou a dabigatrana (em dose fixa de $150 \mathrm{mg}$ duas vezes ao dia, sem controle laboratorial) com varfarina (RNI alvo entre 2-3), demons-

Quadro 23 - Classificação dos novos anticoagulantes por seu mecanismo de ação.

\begin{tabular}{|ll|}
\hline Anticoagulantes indiretos & Fármacos \\
Mediados por antitrombina & $\bullet$ Fondaparinux \\
& $\bullet$ Idraparinux \\
Mediado pelo cofator-ll da heparina & $\bullet$ SSR12517E, SR123781A \\
Mediado pela proteína C & $\bullet$ Odiparcil \\
Anticoagulantes Diretos & $\bullet$ ART-123 \\
Fator IXa & - RB006, TTP889 \\
Fator Xa & $\bullet$ Razaxabana \\
& $\bullet$ Otamixabana \\
& $\bullet$ Apixabana \\
& $\bullet$ Rivaroxabana \\
Trombina & $\bullet$ DX9065a, LY517717, YM150, DU176b, PRT054021 \\
& $\bullet$ Dabigatrana \\
& $\bullet$ Pegmusirudina \\
& $\bullet$ Flovagatrana \\
\hline
\end{tabular}

Adaptado do estudo de Weitz et al. ${ }^{(30)}$ 
trando igual eficácia e segurança no tratamento de TEV aguda. ${ }^{(28)}$ Essa é uma primeira evidência consistente para o seu uso na prática clínica, mas é prudente observar os relatos de farmacovigilância, bem como estudos posteriores que corroborem esses resultados. Apesar de não ter sido testada no contexto de $\mathrm{TlH}$, como no Brasil não são disponíveis fármacos alternativos as heparinas, talvez possa já ser utilizado nessa indicação.

\section{Bivalirudina}

É um polipetídeo semissintético, diferindo da hirudina por ser um inibidor de menor afinidade, promovendo apenas a inibição temporária da trombina. Sua meia-vida é mais curta, resultando em um perfil de segurança mais favorável. Foi mais testado na cardiopatia isquêmica, sendo seu uso em TEV apresentado apenas em relatos de caso de $\mathrm{TlH}^{(29)}$

\section{Inibidores do fator Xa (pentassacarídeos sintéticos)}

Os inibidores do fator Xa podem agir diretamente, ligando-se ao sitio ativo de ação, ou indiretamente, catalisando a ação da antitrombina.

\section{Fondaparinux}

É um pentassacarídeo sintético análogo da heparina, uma espécie de heparina símile, inibidor seletivo do fator $\mathrm{X}$, não se ligando às plaquetas ou ao fator plaquetário 4 . Sua grande limitação é a não reversibilidade pelo sulfato de protamina. A sua eficácia tem sido demonstrada em pacientes com maior risco de eventos tromboembólicos e submetidos a cirurgias orto- pédicas. Houve uma diminuição de 50-60\% desses eventos, e apresentou maior eficácia do que a enoxaparina. ${ }^{(30)}$ Um estudo envolvendo 2.213 pacientes com TEP comparou a eficiência da fondaparinux vs. heparina convencional. A incidência de recidiva foi igual nos dois grupos, e não houve diferenças significativas quanto à incidência de sangramentos maiores, trombocitopenia ou morte. ${ }^{(31)}$

\section{Idraparinux}

Ainda se encontra em estudo, e é um análogo da fondaparinux. Tem ação mais prolongada e pode ser administrado por via s.c. uma vez por semana. ${ }^{(32)}$ Estudos iniciais demonstraram um aumento da taxa de sangramento e uma maior recorrência de TEV nos primeiros 10 dias, sendo, portanto, recomendado iniciar com HBPM na fase aguda e continuar com idraparinux. Como vantagem, há a possibilidade de reversão do efeito anticoagulante com um antídoto específico (avidina).

\section{Razaxabana}

É um pentassacarídeo que poderá ser usado por via oral. Resultados preliminares de um estudo randomizado em fase 11 mostram sua maior eficácia do que a enoxaparina na prevenção de TVP em cirurgias ortopédicas, mas têm-se observado hemorragias mais importantes, principalmente no local da cirurgia. ${ }^{(33)}$

\section{Apixabana}

É uma variante da razaxabana com vantagens farmacocinéticas (maior biodisponibilidade e meia-vida mais longa). Um ensaio clínico para

Quadro 24 - Doses recomendadas de fármacos alternativos para uso em TEV.

\begin{tabular}{|c|c|c|}
\hline Fármacos & Dose terapêutica & Dose profilática \\
\hline Danaparoide & $1.500-2.250$ Ul s.c. a cada $12 \mathrm{~h}$ & 750 Ul s.c. a cada $8-12$ h \\
\hline Argatrobana & $2 \mu \mathrm{g} / \mathrm{kg} / \mathrm{min}$ i.v. continua & NA \\
\hline Lepirudina & $\begin{array}{c}0,4 \mathrm{mg} / \mathrm{kg} \text { i.v. em bolus, seguido de } 0,15 \mathrm{mg} / \mathrm{kg} / \mathrm{h} \text { i.v. continua } \\
\text { ou }\end{array}$ & $25 \mathrm{mg}$ s.c. a cada $12 \mathrm{~h}$ \\
\hline & 0,75-2 mg/kg s.c. cada $12 \mathrm{~h}$ & \\
\hline Fondaparinux & $\begin{array}{l}\text { 50-100 kg de peso: } 7,5 \mathrm{mg} \text { s.c. a cada } 24 \mathrm{~h} \\
<50 \mathrm{~kg} \text { de peso: } 5 \mathrm{mg} \text { s.c. a cada } 24 \mathrm{~h} \\
>100 \mathrm{~kg} \text { de peso: } 10 \mathrm{mg} \text { s.c. a cada } 24 \mathrm{~h}\end{array}$ & 2,5 mg s.c. a cada 24 h \\
\hline Dabigatrana & $150 \mathrm{mg}$ v.o. a cada $12 \mathrm{~h}$ & $\begin{array}{l}110 \mathrm{mg} \text { v.o. } 1-4 \mathrm{~h} \text { após a cirurgia } \\
\text { No dia seguinte, } 220 \mathrm{mg} \text { v.o. a } \\
\text { cada } 24 \mathrm{~h}\end{array}$ \\
\hline
\end{tabular}

NA: Não avaliado. Adaptado de estudos anteriores..$^{(9,30)}$ 
profilaxia do TEV em pacientes ortopédicos comparando o uso de apixabana (doses diárias 5 e $10 \mathrm{mg} / \mathrm{dia}$, em regimes de uma ou duas vezes ao dia) com o de varfarina ou enoxaparina demonstrou uma redução da incidência de TEV e da mortalidade total com todas as doses. ${ }^{(34)} \mathrm{Na}$ dose diária de $5 \mathrm{mg} \mathrm{(2,5} \mathrm{mg} \mathrm{duas} \mathrm{vezes} \mathrm{ou} 5 \mathrm{mg}$ uma vez), houve menor incidência de sangramento. Estudos para tratamento de TEV estão em andamento.

\section{Rivaroxabana}

É um derivado da oxazolidona que inibe o fator Xa e tem excelente biodisponibilidade oral e meia-vida de 9 h. ${ }^{(29)}$ Um grande ensaio clinico em pacientes submetidos a cirurgias ortopédicas maiores comparando o uso de rivaroxabana (10 mg/dia, v.o., iniciado 6-8 h após a cirurgia) com o de enoxaparina s.c. (40 mg/dia, iniciada na noite anterior), demonstrou uma redução em 49\% no desfecho combinado de TEV e mortalidade, sem aumento no risco de sangramento. ${ }^{(35)}$ A rivaroxabana também foi avaliado para o tratamento de TVP proximal através de estudos de dose-resposta (fase 11), os quais verificaram uma redução da progressão do trombo e de desfechos clínicos, mas sem relação com a dose do fármaco. ${ }^{(36)}$

\section{Outros fármacos anticoagulantes}

Alguns novos fármacos estão sendo testados em estudos experimentais e em ensaios clínicos de fase 11 para a profilaxia de TEV, entre eles os inibidores da propagação da coagulação (DPC 906, trombomodulina solúvel) e os moduladores da atividade fibrinolítica endógena (inibidores de PAl-1, inibidores TAFla e inibidores do fator XIlla). Aguardam-se os resultados de ensaios clínicos de fase 111 para se definir o seu papel na prática assistencial. ${ }^{(5)}$

As doses recomendadas para os fármacos alternativos estão descritos no Quadro 24.

As recomendações são as seguintes:

1) Fármacos alternativos, como danaparoide, hirudina recombinante $\mathrm{e}$ fondaparinux, podem ser utilizados nos casos de trombocitopenia autoimune induzida por heparina.(C)

2) Os resultados dos ensaios clínicos disponíveis não permitem recomendar esses novos fármacos como tratamento de eleição em substituição das heparinas e cumarínicos.(D)
3) Tendo em vista a não disponibilidade da maioria desses fármacos no mercado brasileiro, o seu uso assistencial deverá ser autorizado pela ANVISA e no âmbito de projetos de pesquisa aprovados pela Comissão Nacional de Ética em Pesquisa.(D)

\section{Referências}

1. Hyers TM. Management of venous thromboembolism: past, present, and future. Arch Intern Med. 2003;163(7):759-68.

2. Walenga JM, Bick RL. Heparin-induced thrombocytopenia, paradoxical thromboembolism, and other side effects of heparin therapy. Med Clin North Am. 1998;82(3):635-58.

3. Crowther MA, Ginsberg JS, Tollefsen DM, Blinder MA. Heparin. In: Hoffman R, Benz E, Shattil SJ, Furie $\mathrm{B}$, Cohen $\mathrm{H}$, et al. Hematology basic principles and practice. Philadelphia: Churchill Livingstone; 2000. p. 2046-56.

4. Eitzman DT, Chi L, Saggin L, Schwartz RS, Lucchesi BR, Fay WP. Heparin neutralization by plateletrich thrombi. Role of platelet factor 4. Circulation. 1994;89(4):1523-9.

5. Weitz Jl, Hirsh J, Samama MM. New anticoagulant drugs: the Seventh ACCP Conference on Antithrombotic and Thrombolytic Therapy. Chest. 2004;126(3 Suppl):265S$286 \mathrm{~S}$.

6. Warketin TE. Danaparoid (Orgaran ${ }^{\circledR}$ ) for the treatment of heparin-induced thrombocytopenia (HIT) and thrombosis: effects on in vivo thrombin and crosslinked fibrin generation, and evaluation of the clinic significance on in vivo cross-reactivity (XR) of danaparoid for HIT-lgG [abstract]. Blood. 1996;88(Suppl 1):626A.

7. Chong BH, Gallus AS, Cade JF, Magnani H, Manoharan A, Oldmeadow M, et al. Prospective randomised openlabel comparison of danaparoid with dextran 70 in the treatment of heparin-induced thrombocytopaenia with thrombosis: a clinical outcome study. Thromb Haemost. 2001;86(5):1170-5.

8. Ibbotson T, Perry CM. Danaparoid: a review of its use in thromboembolic and coagulation disorders. Drugs. 2002;62(15):2283-314.

9. Hassell K. The management of patients with heparininduced thrombocytopenia who require anticoagulant therapy. Chest. 2005;127(2 Suppl):1S-8S.

10. Mosesson MW. Fibrin polymerization and its regulatory role in hemostasis. J Lab Clin Med. 1990;116(1):8-17.

11. Greinacher A, Völpel H, Janssens U, Hach-Wunderle V, Kemkes-Matthes B, Eichler P, et al. Recombinant hirudin (lepirudin) provides safe and effective anticoagulation in patients with heparin-induced thrombocytopenia: a prospective study. Circulation. 1999;99(1):73-80.

12. Tsuda Y, Szewczuk Z, Wang J, Yue SY, Purisima E, Konishi Y. Interactions of hirudin-based inhibitor with thrombin: critical role of the lleH59 side chain of the inhibitor. Biochemistry. 1995;34(27):8708-14.

13. Kelton JG. The clinical management of heparin-induced thrombocytopenia. Semin Hematol. 1999;36(1 Suppl 1):17-21.

14. Greinacher A, Eichler P, Albrecht D, Strobel U, Pötzsch B, Eriksson Bl. Antihirudin antibodies following low-dose subcutaneous treatment with desirudin for thrombosis prophylaxis after hip-replacement surgery: incidence and clinical relevance. Blood. 2003;101(7):2617-9. 
15. Lubenow N, Eichler P, Lietz T, Greinacher A; Hit Investigators Group. Lepirudin in patients with heparininduced thrombocytopenia - results of the third prospective study (HAT-3) and a combined analysis of HAT-1, HAT-2, and HAT-3. J Thromb Haemost. 2005;3(11):2428-36.

16. Fischer KG, Liebe V, Hudek R, Piazolo L, Haase KK, Borggrefe $\mathrm{M}$, et al. Anti-hirudin antibodies alter pharmacokineticsand pharmacodynamics of recombinant hirudin. Thromb Haemost. 2003;89(6):973-82.

17. ClarkeRJ, Mayo G, FitzGerald GA, Fitzgerald DJ. Combined administration of aspirin and a specific thrombin inhibitor in man. Circulation. 1991;83(5):1510-8.

18. Sheth SB, DiCicco RA, Hursting MJ, Montague T, Jorkasky DK. Interpreting the International Normalized Ratio (INR) in individuals receiving argatroban and warfarin. Thromb Haemost. 2001;85(3):435-40.

19. Swan SK, Hursting MJ. The pharmacokinetics and pharmacodynamics of argatroban: effects of age, gender, and hepatic or renal dysfunction. Pharmacotherapy. 2000;20(3):318-29.

20. Matthai WH Jr, Hursting MJ, Lewis BE, Kelton JG. Argatroban anticoagulation in patients with a history of heparin-induced thrombocytopenia. Thromb Res. 2005;116(2):121-6.

21. Institute for Clinical System Improvement. Anticoagulant Therapy Supplement. Bloomington: Institute for Clinical System Improvement; 2001.

22. Hirsh J, Weitz Jl. New antithrombotic agents. Lancet. 1999;353(9162):1431-6. Erratum in: Lancet. 1999;353(9166):1804.

23. Shapiro SS. Treating thrombosis in the 21 st century. N Engl J Med. 2003;349(18):1762-4.

24. Gurewich V. Ximelagatran--promises and concerns. JAMA. 2005;293(6):736-9.

25. Eriksson Bl, Dahl OE, Büller HR, Hettiarachchi R, Rosencher N, Bravo ML, et al. A new oral direct thrombin inhibitor, dabigatran etexilate, compared with enoxaparin for prevention of thromboembolic events following total hip or knee replacement: the BISTRO 11 randomized trial. J Thromb Haemost. 2005;3(1):103-11.

26. Eriksson Bl, Dahl OE, Rosencher N, Kurth AA, van Dijk CN, Frostick SP, et al. Oral dabigatran etexilate vs. subcutaneous enoxaparin for the prevention of venous thromboembolism after total knee replacement: the RE-MODEL randomized trial. J Thromb Haemost. 2007;5(11):2178-85.

27. Eriksson Bl, Dahl OE, Rosencher N, Kurth AA, van Dijk CN, Frostick SP, et al. Dabigatran etexilate versus enoxaparin for prevention of venous thromboembolism after total hip replacement: a randomised, double-blind, non-inferiority trial. Lancet. 2007;370(9591):949-56. Erratum in: Lancet. 2007;370(9604):2004.
28. Schulman S, Kearon C, Kakkar AK, Mismetti P, Schellong $\mathrm{S}$, Eriksson $\mathrm{H}$, et al. Dabigatran versus warfarin in the treatment of acute venous thromboembolism. N Engl J Med. 2009;361(24):2342-52.

29. Weitz Jl, Hirsh J, Samama MM; American College of Chest Physicians. New antithrombotic drugs: American College of Chest Physicians Evidence-Based Clinical Practice Guidelines (8th Edition). Chest. 2008;133(6 Suppl):234S-256S. Erratum in: Chest. 2008;134(2):473.

30. Turpie AG, Bauer KA, Eriksson Bl, Lassen MR; PENTATHALON 2000 Study Steering Committee. Postoperative fondaparinux versus postoperative enoxaparin for prevention of venous thromboembolism after elective hip-replacement surgery: a randomised double-blind trial. Lancet. 2002;359(9319):1721-6. Erratum in: Lancet. 2002;360(9339):1102.

31. Büller HR, Davidson BL, Decousus H, Gallus A, Gent M, Piovella F, et al. Subcutaneous fondaparinux versus intravenous unfractionated heparin in the initial treatment of pulmonary embolism. $\mathrm{N}$ Engl $\mathrm{J}$ Med. 2003;349(18):1695-702. Erratum in: N Engl J Med. 2004;350(4):423.

32. PERSIST investigators. A novel long-acting synthetic factor Xa inhibitor (SanOrg34006) to replace warfarin for secondary prevention in deep vein thrombosis. A Phase 11 evaluation. J Thromb Haemost. 2004;2(1):4753. Erratum in: J Thromb Haemost. 2004;2(3):540.

33. Lassen MR, Davidson BL, Gallus A, et al. A phase 11 randomized, double-blind, five-arm, parallel-group, dose-response study of a new oral directly acting factor Xa inhibitor, razaxaban, for the prevention of deep vein thrombosis in knee replacement surgery - on behalf of the Razaxaban investigators [abstract]. Blood. 2003;102(Suppl 1):15A

34. Lassen MR, Davidson BL, Gallus A, Pineo G, Ansell J, Deitchman D. The efficacy and safety of apixaban, an oral, direct factor $\mathrm{X}$ a inhibitor, as thromboprophylaxis in patients following total knee replacement. J Thromb Haemost. 2007;5(12):2368-75.

35. Lassen MR, Turpie AG, Rosencher N, Borris LC, Ageno $\mathrm{W}$, Lieberman JR, et al. Late breaking clinical trial: rivaroxaban, an oral, direct factor $\mathrm{Xa}$ inhibitor, for the prevention of venous thromboembolism in total knee replacement surgery: results of the RECORD-3 study-late breaking. J Thromb Haemost. 2007;5(Suppl 2):0-S-006B.

36. Buller HR, EINSTEIN-DVT Study Group. Once-daily treatment with an oral, direct factor $X_{a}$ inhibitor, rivaroxaban (BAY 59-7939), in patients with acute, symptomatic deep vein thrombosis: the EINSTEIN-DVT dose-finding study. Eur Heart J. 2006;27(Suppl 1):761A. 